\title{
Polaronic Mechanism of Superconductivity in Cuprates
}

\author{
Pradeep Chaudhary, ${ }^{1}$ Anuj Nuwal, ${ }^{2}$ S. C. Tiwari, ${ }^{3}$ R. K. Paliwal, ${ }^{4}$ and S. L. Kakani ${ }^{5}$ \\ ${ }^{1}$ Department of Physics, Government Polytechnic College, Chittorgarh, Rajasthan 312001, India \\ ${ }^{2}$ Department of Physics, Sangam University, N.H. No. 79, Bhilwara By-Pass, Chittor Road, Bhilwara, Rajasthan 311001, India \\ ${ }^{3}$ Department of Physics, M.L.V. Government College, Bhilwara, Rajasthan 311001, India \\ ${ }^{4}$ Department of Physics, Mewar University, Gangrar, Chittorgarh, Rajasthan 312901, India \\ ${ }^{5}$ 4-G-45, Shastri Nagar, New Housing Board, Bhilwara, Rajasthan 311001, India
}

Correspondence should be addressed to S. L. Kakani; slkakani28@gmail.com

Received 15 April 2013; Accepted 13 June 2013

Academic Editor: Zigang Deng

Copyright (C) 2013 Pradeep Chaudhary et al. This is an open access article distributed under the Creative Commons Attribution License, which permits unrestricted use, distribution, and reproduction in any medium, provided the original work is properly cited.

\begin{abstract}
A strong polaron pairing model of high-temperature cuprate superconductors is presented. The normal and anomalous oneparticle Green's functions are derived from a system with strong electron-phonon coupling. Self-consistent equation for the superconducting order parameter $(\Delta)$ is derived using Green's function technique and following Lang and Firsov transformations. Expressions for specific heat, density of states, free energy, and critical field based on this model have been derived. The theory is applied to explain the experimental results in the system $\mathrm{YBa}_{2} \mathrm{Cu}_{3} \mathrm{O}_{7-x}$. There is convincing evidence that the theory is fully compatible with the key experiments.
\end{abstract}

\section{Introduction}

Strikingly, after 26 years of enormous experimental and theoretical efforts followed by the discovery, there is still little consensus on the pairing mechanism of high-temperature superconductivity (HTSC) in cuprates [1-12]. HTSCs have unique physical properties in both the normal state and superconducting one. To comprehend the physics of these complex compounds is one of the main tasks of the theory of superconductivity, whose solution may allow one to explain the pairing mechanism ensuring HTSC. At present, there exists no mechanism which would explain the totality of thermodynamical, magnetic, and superconductive properties of HTSCs from a single point of view.

The electron-phonon pairing mechanism [13-16], being the principal one in low-temperature superconductors, makes a considerable contribution to the establishment of the superconducting state in HTSCs. But in order to obtain proper description, it is necessary to consider the other mechanism inherent in HTSCs $[3,5,7,8,10]$.

To explain HTSC, a lot of models and mechanisms of this unique phenomenon have been proposed $[1-5,10$,
11]. The key question is the nature of the mechanism of pairing of carriers. There are many different models of superconductivity available, for example, magnon model, exciton model, model of resonant valence bonds, bipolaronic model, bisoliton model, anharmonic model, model of local pairs, and plasmon model, [17]. All these models use the concept of pairing with a subsequent formation of a Bose condensate at temperature $T_{C}$ irrespective of the nature of the resulting attraction. Some recent theoretical models postulate the mechanism of antiferromagnetic spin fluctuations [18, 19], so that the electron scattering on them can be the reason for the pairing of electrons.

In order to comprehend the nature of the superconducting state, it is necessary to construct a consistent microscopic theory which should be able to describe superconductive and the normal properties of HTSCs. We have proposed that the pairing mechanism in cuprate superconductors can be understood on the polaronic model of the charge carriers [20-22].

In the present work following Alexandrov and Ranninger [21], we have developed a microscopic theory for HTSC cuprates by generalizing the Holstein [22] and Lang Firsov 
$[23,24]$ and Gorkov Nambu formalisms in order to evaluate the Green's functions for electrons coupled to phonons and considering the range of coupling which corresponds to small polaron formation $(\lambda \gg 1)[25,26]$.

\section{Model Hamiltonian}

The model Hamiltonian for our system can be expressed as [21]

$$
H=H_{e}+H_{\mathrm{ph}}+H_{e-\mathrm{ph}}+H_{e e},
$$

where $H_{e}$ is the kinetic energy in the initial Bloch band, $H_{\mathrm{ph}}$ is the vibration energy of the lattice, $H_{e-\mathrm{ph}}$ is electron-phonon interaction, and $H_{e e}$ is the Coulomb electron-electron correlations.

In one band approximation $H_{e}$ has the form

$$
H_{e}=\sum_{k s} E(k) C_{k s}^{+} C_{k s}
$$

where $k$ and $s$ denote the state with quasi momentum and spin, respectively. $E(k)$ is bare band energy. $H_{\mathrm{ph}}$ can be expressed in terms of phonon operators $d_{q} ; q=(q, v)$ where $v$ is the type of vibrational mode

$$
H_{\mathrm{ph}}=\sum_{q} \omega(q) d_{q}^{+} d_{q}
$$

where $\omega$ is the phonon dispersion.

The electron-phonon interaction is described by the Fröhlich Hamiltonian:

$$
H_{e-\mathrm{ph}}=\sum_{k, q, s}\left\{\omega(q) \gamma(q) \frac{1}{\sqrt{2 N}} C_{k+q, s}^{+} C_{k, s} d_{q}+\text { H.C. }\right\}
$$

in which $\omega(q)$ and $\gamma(q)$ are the phonon frequency and the interaction matrix element in a parent crystal without charge carriers, respectively. Correspondingly one obtains

$$
\gamma^{2}(q)=\frac{4 \pi e^{2}}{q^{2} \Omega \omega_{0}}\left(\frac{1}{\varepsilon_{\infty}}-\frac{1}{\varepsilon_{0}}\right) .
$$

In the case of optical longitudinal phonon with frequency $\omega(q)=\omega_{0}$ and $\varepsilon_{0}, \varepsilon_{\infty}$ are the dielectric constants of the crystal with and without taking ionic part into consideration. $\Omega$ is the volume of the unit cell and $N$ is their number.

For acoustic phonons, one finds

$$
\begin{gathered}
v^{2}(q)=E_{D}^{2} \frac{q}{u M}, \\
\omega(q)=\omega_{0},
\end{gathered}
$$

where $E_{D}$ is the deformation potential, $u$ is the sound velocity, and $M$ is the mass of an elementary cell. For intermolecular phonons

$$
\gamma^{2}(q)=\gamma^{2}(0)
$$

The combined Hamiltonian can be expressed as

$$
\begin{aligned}
H= & \sum_{k s} E(k) C_{k s}^{+} C_{k s}+\sum_{q} \omega(q) d_{q}^{+} d_{q} \\
& +\sum_{k, q, s} \omega(q) \gamma(q) d_{q} \frac{1}{\sqrt{2 N}}\left\{C_{k+q}^{+} C_{k, s}+H . C .\right\}+V_{C} .
\end{aligned}
$$

Here $V_{C}$ is the Coulomb repulsion. This Hamiltonian includes electron-phonon and electron-electron correlations. To diagonalize the main part of the Hamiltonian, the site representation is more convenient.

One can express the previous Hamiltonian as

$$
\begin{aligned}
H= & \sum_{m n} T(m-n) C_{m \sigma}^{+} C_{n \sigma}+\sum_{q} \omega(q)\left\{d_{q}^{+} d_{q}+\frac{1}{2}\right\} \\
& +\frac{1}{\sqrt{2 N}} \sum_{m q} \omega(q) \gamma(q) n_{m \sigma}\left\{d_{q} e^{i q m}+d_{q}^{+} e^{-i q m}\right\} \\
& +\frac{1}{2} \sum_{m n \sigma \sigma^{\prime}} V_{C}(m-n) n_{m \sigma}^{+} n_{n \sigma^{\prime}}+\sum_{q} \omega(q)\left\{d_{q}^{+} d_{q}+\frac{1}{2}\right\},
\end{aligned}
$$

where

$$
T(m)=\frac{1}{N} \sum_{k} E(k) e^{i k m}, \quad n_{m \sigma}=C_{m \sigma}^{+} C_{m \sigma} .
$$

In the small-polaron regime, $\lambda \geq 1$, the kinetic energy remains smaller than the interaction energy, and a selfconsistent treatment of a many-body problem is possible with the $1 / \lambda$ expansion technique [27]. Following Lang and Firsov $[23,24]$ and applying canonical transformations to diagonalize the Hamiltonian [28], one obtains

$$
\widetilde{H}=e^{S} H e^{-S},
$$

where

$$
S=\sum_{m s} C_{m s}^{+} C_{m s} \frac{1}{\sqrt{2 N}} \gamma(q)\left\{d_{q} e^{i q m}-d_{q}^{+} e^{-i q m}\right\} .
$$

The electron operator transforms as

$$
\begin{gathered}
\tilde{d}_{q}=d_{q^{\prime}}+\left[S, d_{q^{\prime}}\right] \frac{1}{2}\left[S,\left[S, d_{q^{\prime}}\right]\right]+\cdots, \\
{\left[s, d_{q^{\prime}}\right]=\left[\sum_{m s} C_{m s}^{+} C_{m s} \frac{1}{\sqrt{2 N}} \gamma(q)\left\{d_{q} e^{i q m}-d_{q}^{+} e^{-i q m}\right\}, d_{q^{\prime}}\right],} \\
{\left[s, d_{q^{\prime}}\right]=\frac{1}{\sqrt{2 N}} \gamma(q)} \\
\times\left[\sum _ { q m s } C _ { m s } ^ { + } C _ { m s } \left\{d_{q} e^{i q m} d_{q^{\prime}}-d_{q}^{+} e^{-i q m} d_{q^{\prime}}\right.\right. \\
\left.\left.-d_{q^{\prime}} d_{q} e^{i q m}+d_{q^{\prime}} d_{q}^{+} e^{-i q m}\right\}\right] .
\end{gathered}
$$


Using $d_{q^{\prime}} d_{q}^{+}=d_{q}^{+} d_{q^{\prime}}+\delta_{q q^{\prime}}$

$$
\left[s, d_{q^{\prime}}\right]=\frac{1}{\sqrt{2 N}} \gamma(q)\left[\sum_{q m s} C_{m s}^{+} C_{m s} \delta_{q q^{\prime}} e^{-i q m}\right] .
$$

When $q=q^{\prime}$ we have

$$
\begin{gathered}
{\left[s, d_{q^{\prime}}\right]=\frac{1}{\sqrt{2 N}} \gamma(q)\left[\sum_{m s} C_{m s}^{+} C_{m s} e^{-i q^{\prime} m}\right]} \\
\left\lfloor s,\left\lfloor s, d_{q^{\prime}}\right\rfloor\right\rfloor=0 .
\end{gathered}
$$

Thus,

$$
\begin{aligned}
& \widetilde{d}_{q^{\prime}}=d_{q^{\prime}}+\frac{1}{\sqrt{2 N}} \gamma(q) \sum_{m s} C_{m s}^{+} C_{m s} e^{-i q^{\prime} m}, \\
& \widetilde{d}_{q^{\prime}}^{+}=d_{q^{\prime}}^{+}-\frac{1}{\sqrt{2 N}} \gamma(q) \sum_{m s} C_{m s}^{+} C_{m s} e^{-i q^{\prime} m}
\end{aligned}
$$

Hence

$$
\begin{aligned}
H= & \sum_{m n s, m \neq n} T(m-n) C_{m s}^{+} C_{n s} \\
& \times \exp \left[\sum_{q} d_{q}\left\{\frac{1}{\sqrt{2 N}} \gamma(q) e^{i q m}\right\}+\sum_{q} \omega(q)\left(d_{q}^{+} d_{q}+\frac{1}{2}\right)\right] \\
& +\sum_{m n s}\left\{V_{C}(m-n)-\sum_{q} \omega(q) \frac{1}{2 N} \gamma^{2}(q) e^{i q(m-n)}\right\} \\
& \times C_{m s}^{+} C_{n s}^{+} C_{n s} C_{m s} .
\end{aligned}
$$

In obtaining (17), we have omitted the term containing the on-site interaction $m=n$ for parallel spins.

\section{Green's Functions}

We define the following one particle temperature electron $(G)$ and anomalous $(F)$ Green's functions:

$$
\begin{aligned}
& G\left(k, \omega_{n}\right)=-\frac{1}{2} \sum_{m} \int_{-\beta}^{\beta} \mathrm{d} l e^{l \omega} n e^{+i k m}\left\langle\left\langle l_{l} C_{c \sigma}(l) C_{m \sigma}^{+}(o)\right\rangle\right\rangle, \\
& F\left(k, \omega_{n}\right)=-\frac{1}{2} \sum_{m} \int_{-\beta}^{\beta} \mathrm{d} l e^{l \omega} n e^{+i k m}\left\langle\left\langle l_{l} C_{c \sigma}(l) C_{m \sigma}(o)\right\rangle\right\rangle .
\end{aligned}
$$

For convenience, dropping spin and applying the LangFirsov canonical transformation and neglecting the residual polaron-polaron coupling and following equation of motion method for the evaluation of electron part and Feynman method for the evaluation of phonon part, one finally obtains [28]

$$
\begin{aligned}
G(t)= & -i e^{\beta \Omega_{e l}} \operatorname{Tr}\left[e^{-i v(m-n) t} C C^{+} e^{-\beta \widetilde{H}_{e l}}\right] \\
& \times \operatorname{Tr}\left[e^{\beta \Omega_{p h}} e^{-\beta \widetilde{H}_{\mathrm{ph}}} X(t) X^{+}(0)\right] .
\end{aligned}
$$

After evaluating the electron part and phonon part of the trace, we obtain the total Green's function as

$$
\begin{aligned}
& G\left(k, \omega_{n}\right) \\
& =e^{-q^{2}}\left[\frac{u_{k}^{2}}{i \omega_{n}-\varepsilon_{n}}+\frac{v_{k}^{2}}{i \omega_{n}+\varepsilon_{n}}+\frac{1}{N} \sum_{l=1}^{\infty} \frac{g^{2 l}}{! l}\right. \\
& \times \sum_{k^{\prime}}\left\{\frac{u_{k^{\prime}}^{2}\left(1-n_{k^{\prime}}\right)}{i \omega_{n}-l \omega_{0}-\varepsilon_{k^{\prime}}}+\frac{v_{k^{\prime}}^{2} n_{k^{\prime}}}{i \omega_{n}-l \omega_{0}+\varepsilon_{k^{\prime}}}\right. \\
& \left.\left.+\frac{u_{k^{\prime}}^{2} n_{k^{\prime}}}{i \omega_{n}+l \omega_{0}-\varepsilon_{k^{\prime}}}+\frac{v_{k^{\prime}}^{2}\left(1-n_{k^{\prime}}\right)}{i \omega_{n}+l \omega_{0}+\varepsilon_{k^{\prime}}}\right\}\right]
\end{aligned}
$$

$$
\begin{aligned}
& F\left(k, \omega_{n}\right) \\
& =e^{-q^{2}}\left[u_{k} v_{k}\left(\frac{1}{i \omega_{n}-\varepsilon_{k}}+\frac{1}{i \omega_{n}+\varepsilon_{k}}\right)+\frac{1}{N} \sum_{l=1}^{\infty} \frac{(-1)^{l} g^{2 l}}{! l}\right. \\
& \times \sum_{k^{\prime}} u_{k^{\prime}} v_{k^{\prime}}\left\{\frac{\left(1-n_{k^{\prime}}\right)}{i \omega_{n}-l \omega_{0}-\varepsilon_{k^{\prime}}}-\frac{n_{k^{\prime}}}{i \omega_{n}-l \omega_{0}+\varepsilon_{k^{\prime}}}\right. \\
& \left.\left.+\frac{n_{k^{\prime}}}{i \omega_{n}+l \omega_{0}-\varepsilon_{k^{\prime}}}-\frac{\left(1-n_{k^{\prime}}\right)}{i \omega_{n}+l \omega_{0}+\varepsilon_{k^{\prime}}}\right\}\right] \text {, }
\end{aligned}
$$

where

$$
\begin{gathered}
u_{k}^{2}=\frac{1}{2}\left(1+\frac{\xi_{k}}{\varepsilon_{k}}\right), \quad v_{k}^{2}=\frac{1}{2}\left(1-\frac{\xi_{k}}{\varepsilon_{k}}\right), \\
u_{k} v_{k}=-\left\{\frac{\Delta}{2 \varepsilon_{k}}\right\}, \quad n_{k}=n\left(\varepsilon_{k}\right) .
\end{gathered}
$$

With

$$
\begin{gathered}
n(x)=\left(e^{x / k_{B} T}+1\right)^{-1}, \\
\varepsilon_{k}=\sqrt{\xi_{k}^{2}+\Delta^{2}(k)}, \\
\Delta(k)=-\frac{1}{2} \sum_{k} V\left(k-k^{\prime}\right) \frac{\Delta\left(k^{\prime}\right)}{\varepsilon_{k^{\prime}}} \tanh \frac{\varepsilon_{k}}{2 k_{B} T}, \\
V(k)=\frac{1}{N} \sum_{m} V(m) e^{i k m} .
\end{gathered}
$$

The energy dispersion for the polaronic band is given by

$$
\xi_{k}=\left\{\sum_{m} \sigma(m o) T(m) e^{i k m}-\mu\right\}
$$

having a narrow band half width $W \ll D$ where $D=$ $Z T(m)\}$. 


\section{Correlation Function}

The correlation functions are defined as

$$
\begin{aligned}
& \left\langle C_{p}^{+} C_{p}\right\rangle=\frac{1}{2 \pi} \int_{-\infty}^{+\infty} I_{G}\left(\omega_{n}\right) d \omega_{n}, \\
& \left\langle C_{p} C_{p}\right\rangle=\frac{1}{2 \pi} \int_{-\infty}^{+\infty} I_{F}\left(\omega_{n}\right) d \omega_{n},
\end{aligned}
$$

where

$$
\begin{gathered}
I_{G}\left(\omega_{n}\right)=i\left(e^{\beta \omega} n+1\right)^{-1}\left[G_{11}\left(\omega_{n}+i \varepsilon\right)-G_{11}\left(\omega_{n}-i \varepsilon\right)\right], \\
I_{F}\left(\omega_{n}\right)=i\left(e^{\beta \omega} n+1\right)^{-1}\left[F_{11}\left(\omega_{n}+i \varepsilon\right)-F_{11}\left(\omega_{n}-i \varepsilon\right)\right],
\end{gathered}
$$

where $G$ and $F$ are Green functions given by (20) and (21), respectively.

Using the identity

$$
\begin{gathered}
\lim _{\varepsilon \rightarrow 0} \frac{1}{2 \pi}\left[\frac{1}{\omega+i \varepsilon-E_{k}}-\frac{1}{\omega-i \varepsilon-E_{k}}\right]=i \delta\left(\omega-E_{k}\right), \\
\quad \int_{-\infty}^{\infty} f\left(\omega_{n}\right) \delta\left(\omega_{n}-\omega^{ \pm}\right) d \omega_{n}=f\left(\omega_{n}^{ \pm}\right) .
\end{gathered}
$$

With the following relations:

$$
u_{k}^{2}=\frac{1}{2}\left(1+\frac{\xi_{k}}{\varepsilon_{k}}\right), \quad v_{k}^{2}=\frac{1}{2}\left(1-\frac{\xi_{k}}{\varepsilon_{k}}\right),
$$

(25) and (26) become

$$
\begin{aligned}
& \left\langle C_{p}^{+} C_{p}\right\rangle \\
& =\frac{1}{2}+\frac{1}{2} \frac{\xi_{k}}{\varepsilon_{k}} \tanh \frac{\beta \varepsilon_{k}}{2} \\
& +\frac{1}{N} \sum_{l=1}^{\infty} \frac{g^{2 l}}{!}\left[\frac{1}{2}+\frac{1}{2} \frac{\xi_{k^{\prime}}}{\varepsilon_{k^{\prime}}} \tanh \frac{\beta\left(l \omega_{0}+\varepsilon_{k^{\prime}}\right)}{2}\right. \\
& \left.\quad-\frac{n_{k^{\prime}}}{2} \frac{\xi_{k^{\prime}}}{\varepsilon_{k^{\prime}}}\left\{\tanh \frac{\beta\left(l \omega_{0}+\varepsilon_{k^{\prime}}\right)}{2}-\tanh \frac{\beta\left(l \omega_{0}-\varepsilon_{k^{\prime}}\right)}{2}\right\}\right], \\
& \left\langle C_{p} C_{p}\right\rangle \\
& =-\frac{\Delta(k)}{2 \varepsilon_{k}} \tanh \frac{\beta \varepsilon_{k}}{2} \\
& \quad+\frac{1}{N} \sum_{l=1}^{\infty} \frac{(-1)^{l}}{! l} g^{2 l} \sum_{k^{\prime}} \frac{\Delta\left(k^{\prime}\right)}{2 \varepsilon_{k^{\prime}}} \\
& \quad \times\left[\tanh \frac{\beta\left(l \omega_{0}+\varepsilon_{k^{\prime}}\right)}{2}\left\{\tanh \frac{\beta\left(l \omega_{0}+\varepsilon_{k^{\prime}}\right)}{2}+\tanh \frac{\beta\left(l \omega_{0}-\varepsilon_{k^{\prime}}\right)}{2}\right\}\right]
\end{aligned}
$$

\section{Superconducting Order Parameter $(\Delta)$}

The order parameter of a superconducting state is given by

$$
\Delta=g \sum_{k}\left\langle C_{k} C_{k}\right\rangle
$$

Substituting correlation function given by (31) in (32) and changing summation into integral using the following relation:

$$
\sum_{k}=N(o) \int_{0}^{\hbar \omega_{D}} d \xi_{k}
$$

the gap equation becomes

$$
\Delta=g N(0) \int_{0}^{\hbar \omega_{D}} d \xi_{k}\left[\frac{-\Delta(k)}{2 \sqrt{\xi_{k}^{2}+\Delta^{2}(k)}} \tanh \frac{\sqrt{\xi_{k}^{2}+\Delta^{2}(k)}}{2 k_{B} T}\right]
$$

$$
-g \frac{1}{N} \sum_{l=1}^{\infty} \frac{(-1)^{l}}{!} g_{1}^{2 l} N(0) \int_{0}^{\hbar \omega_{D}} \sum_{k^{\prime}} d \xi_{k} \frac{-\Delta\left(k^{\prime}\right)}{2 \sqrt{\xi_{k^{\prime \prime}}^{2}+\Delta^{2}\left(k^{\prime}\right)}}
$$

$$
\begin{gathered}
\times\left[\tanh \frac{l \omega_{0}+\sqrt{\xi_{k^{\prime \prime}}^{2}+\Delta^{2}\left(k^{\prime}\right)}}{2 k_{B} T}\right. \\
-\frac{1}{\exp \left\{\sqrt{\left.\xi_{k^{\prime \prime}}^{2}+\Delta^{2}\left(k^{\prime}\right) / 2 k_{B} T\right\}+1}\right.} \\
\times\left\{\tanh \frac{\left(l \omega_{0}+\sqrt{\xi_{k^{\prime \prime}}^{2}+\Delta^{2}\left(k^{\prime}\right)}\right)}{2 k_{B} T}\right. \\
\left.\left.+\tanh \frac{\left(l \omega_{0}-\sqrt{\xi_{k^{\prime \prime}}^{2}+\Delta^{2}\left(k^{\prime}\right)}\right)}{2 k_{B} T}\right\}\right] .
\end{gathered}
$$

Right-hand side of (34) has two terms which are quite independent. First term varies with $k$, whereas second term varies with $k^{\prime}$; hence, one can define two superconducting order parameters for the $\mathrm{YBa}_{2} \mathrm{Cu}_{3} \mathrm{O}_{7-x}$ system. The two independent terms finally yield the two equations as

$$
\frac{1}{g N(0)}=\int_{0}^{\hbar \omega_{D}} d \xi_{k}\left[\frac{1}{2 \sqrt{\xi_{k}^{2}+\Delta^{2}(k)}} \tanh \frac{\sqrt{\xi_{k}^{2}+\Delta^{2}(k)}}{2 k_{B} T}\right]
$$


with $\ell=1$ the other equation is

$$
\begin{aligned}
& \frac{1}{|g| N(0)\left[g_{l}^{2}\right]} \\
& =\int_{0}^{\hbar \omega_{D}} \frac{d \xi_{k^{\prime}}}{2 \sqrt{\xi_{k^{\prime \prime}}^{2}+\Delta^{2}\left(k^{\prime}\right)}}\left[\tanh \frac{\ell \omega_{0}+\sqrt{\xi_{k^{\prime \prime}}^{2}+\Delta^{2}\left(k^{\prime}\right)}}{2 k_{B} T}\right. \\
& -\frac{1}{\exp \left\{\sqrt{\xi_{k^{\prime \prime}}^{2}+\Delta^{2}\left(k^{\prime}\right)} / 2 k_{B} T\right\}+1} \\
& \times\left\{\tanh \frac{\ell \omega_{0}+\sqrt{\xi_{k^{\prime \prime}}^{2}+\Delta^{2}\left(k^{\prime}\right)}}{2 k_{B} T}\right. \\
& \left.\left.+\tanh \frac{\ell \omega_{0}-\sqrt{\xi_{k^{\prime \prime}}^{2}+\Delta^{2}\left(k^{\prime}\right)}}{2 k_{B} T}\right\}\right] .
\end{aligned}
$$

With the help of (35) and (36) one can study the behavior of superconducting order parameters with temperature.

\section{Physical Properties}

6.1. Electronic Specific Heat $\left(c_{e s}\right)$. The electronic specific heat per atom of a superconductor is determined from the following relation $[3,4]$ :

$$
c_{e s}=\frac{\partial}{\partial T}\left[\frac{1}{N} \sum_{k} 2 \xi_{k}\left\langle C_{k}^{+} C_{k}\right\rangle\right],
$$

where $\left\langle C_{k}^{+} C_{k}\right\rangle$ is the correlation function. We have obtained this correlation function in (30). Substituting the correlation function from (30) in equation (37), One obtains

$$
\begin{aligned}
c_{e s}=\frac{N(0)}{2 N} \int_{0}^{\hbar \omega_{D}} \frac{2 \xi_{k}}{k_{B} T^{2}} d \xi_{k} \\
\times\left[-\frac{1}{2} \xi_{k} \sec h^{2}\left(\frac{\varepsilon_{k}}{2 k_{B} T}\right)\right. \\
+\sum_{l=1}^{\infty} \frac{g^{2 l}}{! l}\left\{-\frac{1}{2} \frac{\xi_{k^{\prime}}}{\varepsilon_{k^{\prime}}}\left(\omega_{0} l+\varepsilon_{k^{\prime}}\right)\right. \\
\times \sec h^{2}\left(\frac{\omega_{0} l+\varepsilon_{k^{\prime}}}{2 k_{B} T}\right) \\
+\frac{n_{k^{\prime}}}{2} \frac{\xi_{k^{\prime}}}{\varepsilon_{k^{\prime}}}\left(\omega_{0} l+\varepsilon_{k^{\prime}}\right) \\
\times \sec h^{2}\left(\frac{\omega_{0} l+\varepsilon_{k^{\prime}}}{2 k_{B} T}\right) \\
+\frac{n_{k^{\prime}}}{2} \frac{\xi_{k^{\prime}}}{\varepsilon_{k^{\prime}}}\left(\omega_{0} l-\varepsilon_{k^{\prime}}\right) \\
\left.\left.\times \sec ^{2}\left(\frac{\omega_{0} l-\varepsilon_{k^{\prime}}}{2 k_{B} T}\right)\right\}\right] .
\end{aligned}
$$

Right-hand side of (38) has two terms which are quite independent from each other. First term varies with $k$, whereas second term varies with $k^{\prime}$; hence one can study the behaviour of electronic specific heat of superconductors with temperature.

6.2. Density of States Function $[N(\omega) / N(o)]$. For $\omega>0$, the function can be defined as [5]

$$
N(\omega)=\lim \frac{1}{2 \pi}\left[G_{11}(k, \omega+i \eta)-G_{11}(k, \omega-i \eta)\right] .
$$

Using the following identity:

$$
\lim _{\eta \rightarrow 0} \frac{1}{2 \pi}\left[\frac{1}{\omega+i \eta-\omega_{n}^{+}}-\frac{1}{\omega-i \eta-\omega_{n}^{+}}\right]=i \delta\left(\omega-\omega_{n}^{+}\right)
$$

changing the summation over " $K$ " into an integration, replacing $\varepsilon_{k}$ by $-\varepsilon_{k}$, and combining the terms and using the relations

$$
u_{k}^{2}+v_{k}^{2}=\frac{1}{2}\left(1+\frac{\xi_{k}}{\varepsilon_{k}}\right)+\frac{1}{2}\left(1-\frac{\xi_{k}}{\varepsilon_{k}}\right)=1,
$$

one obtains

$$
\begin{array}{r}
\frac{N(\omega)}{N(0)}=\frac{1}{N} \sum_{l=1}^{l=\infty} \frac{g^{2 l}}{l} \sum_{k^{\prime}}\left\{\frac{i \omega_{n}-l \omega_{0}}{\left\{\left(i \omega_{n}-l \omega_{0}\right)^{2}-\Delta^{2}\right\}^{1 / 2}}\right. \\
\left.-\frac{i \omega_{n}+l \omega_{0}}{\left\{\left(i \omega_{n}+l \omega_{0}\right)^{2}-\Delta^{2}\right\}^{1 / 2}}\right\} .
\end{array}
$$

6.3. Free Energy. It is well known that free energy of normal paramagnetic phase always exceeds the free energy of superconducting diamagnetic phase. The entropy decreases remarkably on cooling the superconductors below the critical temperature. The free energy can easily be defined for the superconducting transition as it is related by the entropy; hence, it also exhibits a similar behavior [3]. Obviously the entropy as well as the free energy difference in the normal state is always greater than the entropy in the superconducting state.

The free energy difference of a superconductor for its normal and superconducting state is given by the following relation [27]:

$$
\frac{F_{s}-F_{N}}{V}=\int_{0}^{\infty} d g\left(\frac{1}{|g|^{2}}\right) \Delta^{2}
$$

where " $g$ " is the interaction parameter and " $\Delta$ " is the superconducting order parameter. Equation (43) can also be expressed as

$$
\frac{F_{s}-F_{N}}{V}=\int_{0}^{\Delta} d \Delta \frac{d}{d \Delta}\left(\frac{1}{|g|^{2}}\right) \Delta^{2}
$$



have

From superconducting order parameter expression, we

$$
\begin{gathered}
\Delta(k)=g N(0) \int_{0}^{\hbar \omega_{D}}\left[\frac{-\Delta(k)}{2 \sqrt{\xi_{k}^{2}+\Delta^{2}(k)}} \tanh \frac{\sqrt{\xi_{k}^{2}+\Delta^{2}(k)}}{2 k_{B} T}\right] d \xi_{k}, \\
\frac{1}{g}=N(0) \int_{0}^{\hbar \omega_{D}}\left[\frac{1}{2 \sqrt{\xi_{k}^{2}+\Delta^{2}(k)}} \tanh \frac{\sqrt{\xi_{k}^{2}+\Delta^{2}(k)}}{2 k_{B} T}\right] d \xi_{k} .
\end{gathered}
$$

Equation (44) becomes

$$
\begin{aligned}
& {\left[\frac{F_{S N}}{V}\right]_{\text {phonon }}} \\
& =\int_{0}^{\Delta(k)} \Delta^{2}(k) d \Delta(k) \frac{d}{d \Delta(k)} \\
& \times\left[N ( 0 ) \int _ { 0 } ^ { \hbar \omega _ { D } } \left\{\frac{1}{2 \sqrt{\xi_{k}^{2}+\Delta^{2}(k)}}\right.\right. \\
& \left.\left.\times \tanh \frac{\sqrt{\xi_{k}^{2}+\Delta^{2}(k)}}{2 k_{B} T}\right\} d \xi_{k}\right] .
\end{aligned}
$$

Since

$$
\begin{gathered}
\varepsilon_{k}^{2}=\xi_{k}^{2}+\Delta^{2}(k), \\
2 \varepsilon_{k} d \varepsilon_{k}=2 \Delta(k) d \Delta(k) .
\end{gathered}
$$

Integrating by parts, we get

$$
\begin{aligned}
2\left[\frac{F_{S N}}{V}\right]= & \frac{\Delta^{2}(k)}{g}-N(0) \\
& \times \int_{0}^{\hbar \omega_{D}} 2 d \xi_{k} \int_{\xi_{k}}^{\varepsilon_{k}} \tanh \left(\frac{\beta \varepsilon_{k}}{2}\right) d \varepsilon_{k}, \\
2\left[\frac{F_{S N}}{V}\right]= & \frac{\Delta^{2}(k)}{g}-\frac{4 N(0)}{\beta} \\
& \times \int_{0}^{\hbar \omega_{D}}\left[\ln \frac{\left\{e^{\beta \varepsilon_{k} / 2}\left(1+e^{-\beta \varepsilon_{k}}\right)\right\}}{\left\{e^{\beta \xi_{k} / 2}\left(1+e^{-\beta \xi_{k}}\right)\right\}}\right] d \xi_{k}, \\
2\left[\frac{F_{S N}}{V}\right]= & \frac{\Delta^{2}(k)}{g}-\frac{4 N(0)}{\beta} \\
& \times \int_{0}^{\hbar \omega_{D}} \ln \left(e^{\beta\left(\varepsilon_{k}-\xi_{k}\right) / 2}\right) d \xi_{k}
\end{aligned}
$$

$$
\begin{gathered}
-\frac{4 N(0)}{\beta} \int_{0}^{\hbar \omega_{D}} \ln \left(1+e^{-\beta \varepsilon_{k}}\right) d \xi_{k} \\
+\frac{4 N(0)}{\beta} \int_{0}^{\hbar \omega_{D}} \ln \left(1+e^{-\beta \xi_{k}}\right) d \xi_{k}
\end{gathered}
$$$$
2\left[\frac{F_{S N}}{V}\right]_{\text {phonon }}
$$$$
=(-)\left[\frac{N(0) \Delta^{2}(k)}{2}-\frac{4 N(0)}{\beta} \frac{e^{-\beta \Delta(k)}}{2}\left(2 \pi \Delta(k) \beta^{-1}\right)^{1 / 2}\right.
$$

$$
\left.+\frac{4 N(0)}{\beta} \frac{1}{\beta}\left(\frac{\pi^{2}}{12}\right)\right] \text {. }
$$

With the help of (49) we can calculate the free energy difference.

6.4. Critical Field $\left(H_{c}\right)$. The critical field is related to the free energy difference as

$$
H_{c}=\left\{8 \pi\left(F_{S}-F_{N}\right)\right\}^{1 / 2} .
$$

Using (49), we obtain

$$
\begin{aligned}
H_{c}=\{8 \pi( & \frac{N(0) \Delta^{2}(k)}{4}-\frac{4 N(0)}{\beta} \\
& \times \frac{e^{-\beta \Delta(k)}}{4}\left(2 \pi \Delta(k) \beta^{-1}\right)^{1 / 2} \\
& \left.\left.+\frac{4 N(0)}{\beta} \frac{1}{\beta}\left(\frac{\pi^{2}}{12}\right)\right)\right\}^{1 / 2} .
\end{aligned}
$$

\section{Numerical Calculations}

Now, we evaluate numerically the physical properties of hightemperature cuprate superconductor $\mathrm{YBa}_{2} \mathrm{Cu}_{3} \mathrm{O}_{7-x}$, using the various relations derived, that is, (35), (36), (38), (42), (49), and (51).

Values of various parameters appearing in the mentioned relations are cited in Table 1. Using these values, we have made study of various parameters related to the physical properties for the system $\mathrm{YBa}_{2} \mathrm{Cu}_{3} \mathrm{O}_{7-x}$.

7.1. Superconducting Order Parameter $(\Delta)$. For the study of superconducting order parameter $(\Delta)$ for the system $\mathrm{YBa}_{2} \mathrm{Cu}_{3} \mathrm{O}_{7-x}$, we have calculated the contributions due to phonons and polarons separately and also obtained the combined effect of phonons and polarons.

(i) Superconducting order parameter $\left(\Delta_{1}\right)$.

(When only electron-phonon interaction is considered). 
TABLE 1: Values of various parameters for HTSC cuprate superconductor for $\mathrm{YBa}_{2} \mathrm{Cu}_{3} \mathrm{O}_{7-x}$.

\begin{tabular}{lll}
\hline S. no. & Property & Value \\
\hline 1 & Superconducting transition temperature $\left(T_{c}\right)$ & $88 \mathrm{~K}$ \\
2 & Density of states $N(0)$ at the Fermi surface & $4.95 \times 10^{26}$ per ergs Cu atom \\
3 & Phonon energy $\hbar \omega_{D}$ & $1.3 \times 10^{-21} \mathrm{~J}$ \\
4 & Polaron frequency $\omega_{\mathrm{pl}}$ & $0.72 \mathrm{eV}$ \\
5 & Polaron density $\left(N(0) \exp \left(g^{2}\right)\right)$ & $8 \mathrm{states} / \mathrm{eV} \mathrm{spin}$ \\
6 & Fermi energy & $0.23 \mathrm{eV}$ \\
7 & Crystal structure & Orthorhombic \\
8 & Cell parameters & $a=0.38 \mathrm{~nm}, b=0.39 \mathrm{~nm}$, and $c=1.17 \mathrm{~nm}$ \\
9 & Number of atoms per unit volume & $5 \times 10^{28} / \mathrm{m}^{3}$ \\
10 & Boltzmann constant $\left(k_{B}\right)$ & $1.38 \times 10^{-23} \mathrm{~J} / \mathrm{K}$ \\
11 & Mass of electron & $9.1 \times 10^{-31} \mathrm{~kg}$ \\
\hline
\end{tabular}

We have (35)

$$
\frac{1}{|g| N(0)}=\int_{0}^{\hbar \omega_{D}} d \xi_{k}\left[\frac{1}{2 \sqrt{\xi_{k}^{2}+\Delta^{2}(k)}} \tanh \frac{\sqrt{\xi_{k}^{2}+\Delta^{2}(k)}}{2 k_{B} T}\right] .
$$

Solving the previous equation numerically, we get

$$
\begin{aligned}
\frac{1}{|g| N(0)}=\int_{y=0}^{y=1} d y & {\left[\frac{1}{2 \sqrt{y^{2}+0.5917 x^{2}}}\right.} \\
& \left.\times \tanh \frac{47.099 \sqrt{y^{2}+0.5917 x^{2}}}{T}\right] .
\end{aligned}
$$

With the help of the previous equation, one can study the variation of superconducting order parameter $\Delta_{1}$ with temperature, when only electron-phonon interaction is considered.

Values of superconducting order parameter obtained at various temperatures are given in Table 2 , and variation of $\Delta_{1}$ with temperature is shown in Figure 1.

(ii) Superconducting order parameter $\left(\Delta_{2}\right)$

(When only polaron interaction is considered)

We have (36)

$$
\begin{aligned}
\frac{1}{g N(0)\left[g_{l}^{2}\right]}=\int_{0}^{\hbar \omega_{D}} & \frac{d \xi_{k^{\prime}}}{2 \sqrt{\xi_{k^{\prime \prime}}^{2}+\Delta^{2}\left(k^{\prime}\right)}} \\
\times & {\left[\tanh \frac{\ell \omega_{0}+\sqrt{\xi_{k^{\prime \prime}}^{2}+\Delta^{2}\left(k^{\prime}\right)}}{2 k_{B} T}\right.}
\end{aligned}
$$

$$
\begin{aligned}
& -\frac{1}{\exp \left\{\sqrt{\xi_{k^{\prime \prime}}^{2}+\Delta^{2}\left(k^{\prime}\right)} / k_{B} T\right\}+1} \\
& \times\left\{\tanh \frac{\ell \omega_{0}+\sqrt{\xi_{k^{\prime \prime}}^{2}+\Delta^{2}\left(k^{\prime}\right)}}{2 k_{B} T}\right.
\end{aligned}
$$

$$
\left.\left.+\tanh \frac{\ell \omega_{0}-\sqrt{\xi_{k^{\prime \prime}}^{2}+\Delta^{2}\left(k^{\prime}\right)}}{2 k_{B} T}\right\}\right]
$$

Solving the previous equation numerically, we get

$$
\begin{aligned}
& \frac{1}{|g| N(0)\left[g_{l}^{2}\right]} \\
& =\int_{y=0}^{y=1} \frac{d y}{2 \sqrt{y^{2}+0.5917 x^{2}}}
\end{aligned}
$$$$
\times\left[\tanh \frac{36.23\left(8+1.3 \sqrt{y^{2}+0.5917 x^{2}}\right)}{T}\right.
$$$$
\begin{aligned}
& -\frac{1}{\exp \left\{94.2 \sqrt{y^{2}+0.5917 x^{2}} / T\right\}+1} \\
& \times\left\{\tanh \frac{36.23\left(8+1.3 \sqrt{y^{2}+0.5917 x^{2}}\right)}{T}\right.
\end{aligned}
$$$$
\left.\left.+\tanh \frac{36.23\left(8-1.3 \sqrt{y^{2}+0.5917 x^{2}}\right)}{T}\right\}\right] .
$$ 
TABLE 2: Superconducting order parameter $(\Delta)$ for $\mathrm{YBa}_{2} \mathrm{Cu}_{3} \mathrm{O}_{7-x}$ system.

\begin{tabular}{|c|c|c|c|c|}
\hline S. no. & $\begin{array}{c}\text { Temperature } \\
(\mathrm{K})\end{array}$ & $\begin{array}{c}\Delta_{1}=x \times 10^{-21} \mathrm{~J} \\
\text { (when only } \\
\text { electron-phonon } \\
\text { interaction is present) }\end{array}$ & $\begin{array}{c}\Delta_{2}=x \times 10^{-21} \mathrm{~J} \\
\text { (when only polaron } \\
\text { interaction is } \\
\text { considered) }\end{array}$ & $\begin{array}{c}\Delta=\Delta_{1}+\Delta_{2}\left(x \times 10^{-21} \mathrm{~J}\right) \\
\text { (in the presence of both } \\
\text { phonon and polaron } \\
\text { interactions) }\end{array}$ \\
\hline 1 & 5 & 2.4869 & 0.114015 & 2.600965 \\
\hline 2 & 10 & 2.4869 & 0.114015 & 2.600965 \\
\hline 3 & 15 & 2.4867 & 0.114015 & 2.600795 \\
\hline 4 & 20 & 2.4865 & 0.114015 & 2.600515 \\
\hline 5 & 25 & 2.4838 & 0.114015 & 2.597865 \\
\hline 6 & 30 & 2.4761 & 0.114015 & 2.590145 \\
\hline 7 & 35 & 2.4600 & 0.114015 & 2.574075 \\
\hline 8 & 40 & 2.4325 & 0.114015 & 2.546595 \\
\hline 9 & 45 & 2.3914 & 0.114015 & 2.505415 \\
\hline 10 & 50 & 2.3340 & 0.114001 & 2.448001 \\
\hline 11 & 55 & 2.2580 & 0.113991 & 2.371991 \\
\hline 12 & 60 & 2.1608 & 0.113970 & 2.274770 \\
\hline 13 & 65 & 2.0382 & 0.113914 & 2.152114 \\
\hline 14 & 70 & 1.8851 & 0.113823 & 1.998923 \\
\hline 15 & 75 & 1.6932 & 0.113683 & 1.806883 \\
\hline 16 & 80 & 1.4457 & 0.113468 & 1.559168 \\
\hline 17 & 81 & 1.3868 & 0.113425 & 1.500225 \\
\hline 18 & 82 & 1.3242 & 0.113370 & 1.437570 \\
\hline 19 & 83 & 1.2571 & 0.113300 & 1.370450 \\
\hline 20 & 84 & 1.1852 & 0.113235 & 1.298435 \\
\hline 21 & 85 & 1.1060 & 0.113177 & 1.219177 \\
\hline 22 & 86 & 1.0190 & 0.113100 & 1.132100 \\
\hline 23 & 87 & 0.9228 & 0.113030 & 1.035830 \\
\hline 24 & 87.9 & 0.8245 & 0.112970 & 0.937470 \\
\hline
\end{tabular}

With the help of the previous equation, one can study the variation of superconducting order parameter $\left(\Delta_{2}\right)$ with temperature, when only polaron interaction is considered.

Values of superconducting order parameter obtained at various temperatures are given in Table 2 , and variation of $\Delta_{2}$ with temperature is shown in Figure 1.

(iii) Superconducting order parameter $\left(\Delta=\Delta_{1}+\Delta_{2}\right)$

(in the presence of combined phonon and polaron interactions)

The superconducting order parameter in the presence of both phonon and polaron interactions can be studied by taking a sum of the order parameters due to phonon and polaron effects. Values of order parameters obtained at various temperatures are given in Table 2.
The behaviour of superconducting order parameter $(\Delta=$ $\Delta_{1}+\Delta_{2}$ ) (combined phonon and polaron interactions) is shown in Figure 1.

7.2. Electronic Specific Heat $\left(C_{e s}\right)$. We have obtained the expression (38) for electronic specific heat putting

$$
\begin{gathered}
\varepsilon_{k}=\left\{\xi^{2}+\Delta^{2}\right\}^{1 / 2}, \\
\Delta(0)=0.5 / \mathrm{eV}, \quad \hbar \omega_{D} \approx 1.3 \times 10^{-21} \mathrm{~J}, \\
\frac{\hbar \omega_{D}}{2 K_{B} T}=47.099 / \mathrm{T} .
\end{gathered}
$$




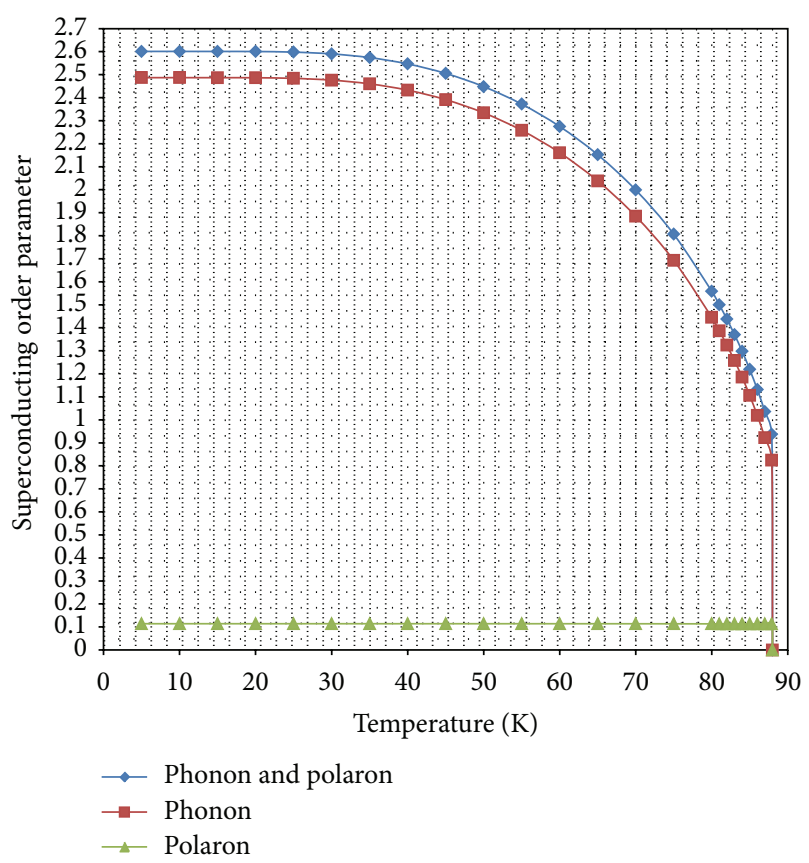

FIGURE 1: Behaviour of superconducting order parameter for the system $\mathrm{YBa}_{2} \mathrm{Cu}_{3} \mathrm{O}_{7-x}$.

Equation (38) reduces to

$$
\begin{aligned}
C_{\mathrm{es}}=2898.5 \times 1.69 \times 10^{-49} \\
\times\left[\int _ { y = 0 } ^ { y = 1 } \frac { y ^ { 2 } } { T ^ { 2 } } d y \left\{-1.3(\operatorname{sech} Q)^{2}\right.\right. \\
-\frac{S}{P}(\operatorname{sech} L)^{2}+\frac{1}{e^{\mathrm{Q}}+1} \frac{S}{P}(\operatorname{sech} L)^{2} \\
\left.\left.-\frac{1}{e^{\mathrm{Q}}+1} \frac{M}{P}(\operatorname{sech} R)^{2}\right\}\right],
\end{aligned}
$$

where

$$
\begin{gathered}
P=\left[y^{2}+0.5917 x^{2}\right]^{1 / 2}, \\
Q=\frac{47.099}{T} P, \\
S=8+1.3 P, \\
M=8-1.3 P, \\
L=\frac{36.23}{T} S, \\
R=\frac{36.23}{T} M .
\end{gathered}
$$

\begin{tabular}{|c|c|c|}
\hline S. no. & $\begin{array}{c}\text { Temperature } \\
(\mathrm{K})\end{array}$ & $\begin{array}{c}C_{\mathrm{es}} \times 10^{-49} \\
\text { Joule/mole-K }\end{array}$ \\
\hline 1 & 87.9 & 127.5236 \\
\hline 2 & 87 & 125.044 \\
\hline 3 & 86 & 122.4406 \\
\hline 4 & 85 & 119.9085 \\
\hline 5 & 84 & 117.4477 \\
\hline 6 & 83 & 115.0882 \\
\hline 7 & 82 & 112.7512 \\
\hline 8 & 81 & 110.4441 \\
\hline 9 & 80 & 108.1521 \\
\hline 10 & 75 & 96.97717 \\
\hline 11 & 70 & 85.96727 \\
\hline 12 & 65 & 74.92361 \\
\hline 13 & 60 & 63.85744 \\
\hline 14 & 55 & 52.85128 \\
\hline 15 & 50 & 42.01019 \\
\hline 16 & 45 & 31.58173 \\
\hline 17 & 40 & 21.92602 \\
\hline 18 & 35 & 13.50073 \\
\hline 19 & 30 & 6.891036 \\
\hline 20 & 25 & 2.565851 \\
\hline 21 & 20 & 0.536428 \\
\hline 22 & 15 & 0.033761 \\
\hline 23 & 10 & 0.000000 \\
\hline 24 & 5 & 0.000000 \\
\hline
\end{tabular}

One can study the behaviour of electronic specific heat $\left(C_{e s}\right)$ with temperature $(T)$ with the help of (57). Values of $C_{e s}$ at various temperatures obtained from (57) are given in Table 3, and variation of $C_{e s}$ with $T$ is shown in Figure 2
TABLE 3: Electronic specific heat $\left(C_{\text {es }}\right)$ for $\mathrm{YBa}_{2} \mathrm{Cu}_{3} \mathrm{O}_{7-x}$ system.

Variation of $C_{\text {es }} / T$ with $T$ (Table 4$)$ and $\left(C-C_{\text {es }}\right) / T$ (Table 5) for a particular range of temperature is shown in Figures 3 and 4, respectively. A comparison with available experimental data is also shown in Figures 3 and 4 [29]. Agreement between theory and experimental results is quite encouraging.

7.3. Density of States Function $N(\omega) / N(0)$. Density of states function for the polaron case is given by

$$
\begin{aligned}
\frac{N(\omega)}{N(0)}=\frac{1}{N} \sum_{l=1}^{l=\infty} \frac{g^{2 l}}{l} \sum_{k^{\prime}}\{ & \frac{i \omega_{n}-l \omega_{0}}{\left\{\left(i \omega_{n}-l \omega_{0}\right)^{2}-\Delta^{2}\right\}^{1 / 2}} \\
& \left.-\frac{i \omega_{n}+l \omega_{0}}{\left\{\left(i \omega_{n}+l \omega_{0}\right)^{2}-\Delta^{2}\right\}^{1 / 2}}\right\} .
\end{aligned}
$$




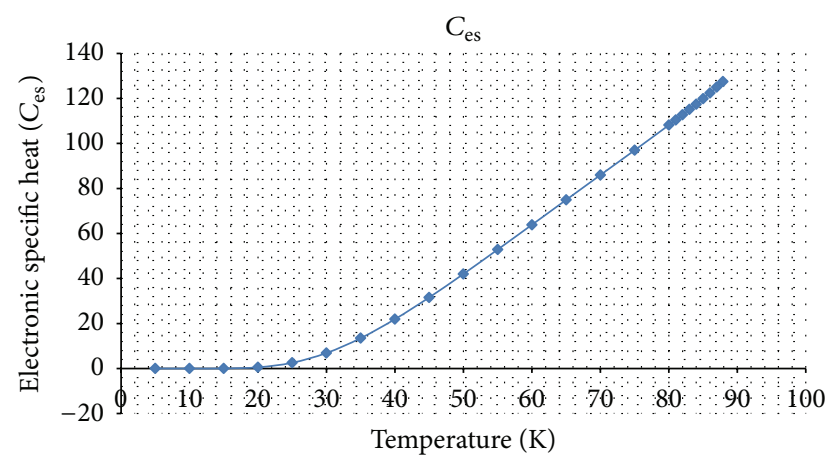

Figure 2: Variation of $C_{\mathrm{es}}$ with temperature for the system $\mathrm{YBa}_{2} \mathrm{Cu}_{3} \mathrm{O}_{7-x}$.

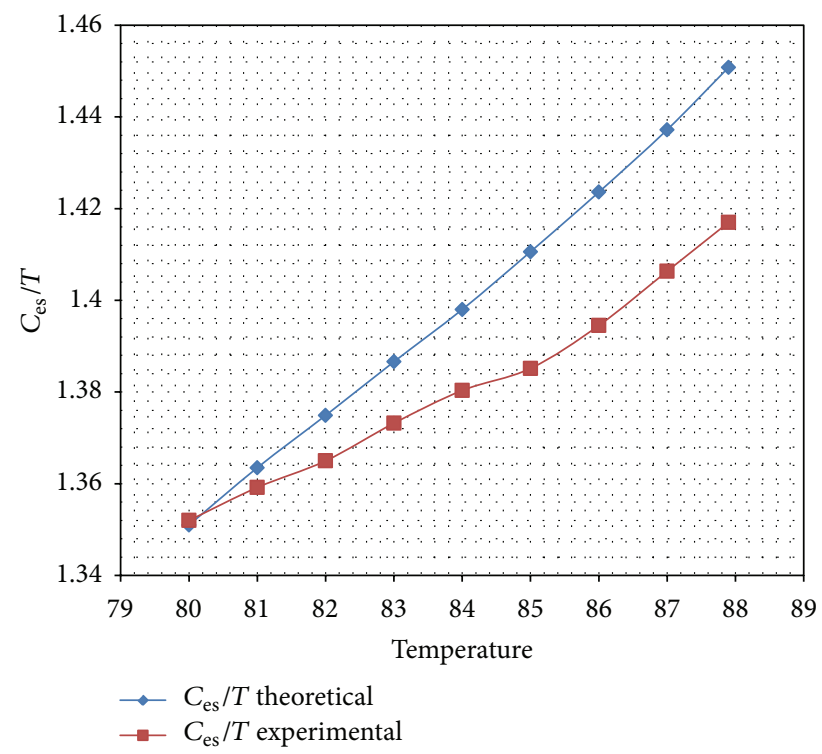

FIgURE 3: Variation of $C_{e s} / T$ with temperature.

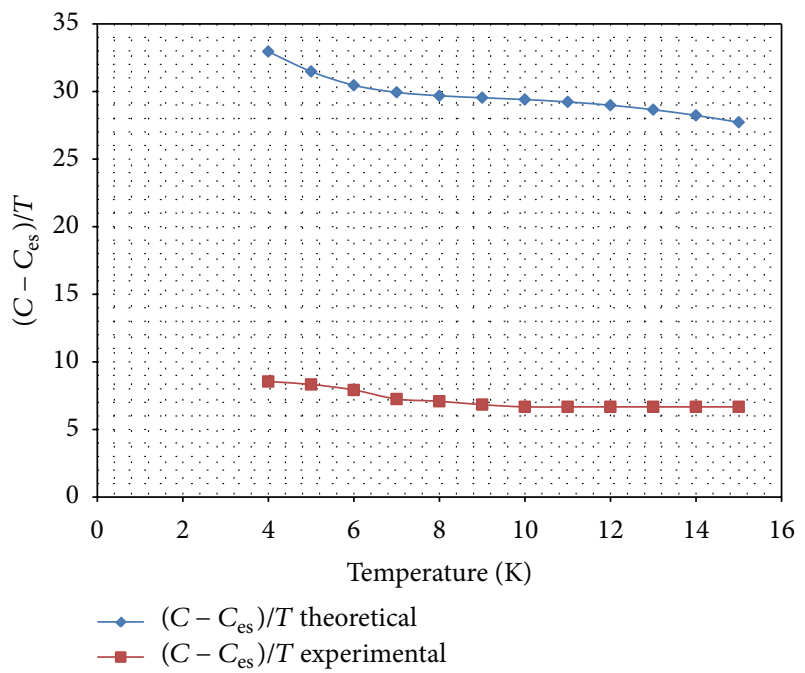

FIgURE 4: Variation of $\left(C-C_{e s}\right) / T$ with temperature.
TABle 4: $C_{\text {es }} / T$ for $\mathrm{YBa}_{2} \mathrm{Cu}_{3} \mathrm{O}_{7-x}$ system.

\begin{tabular}{|c|c|c|c|}
\hline S. no. & $\begin{array}{c}\text { Temperature } \\
(\mathrm{K})\end{array}$ & $\begin{array}{c}C_{\mathrm{es}} / T \\
\text { Joule/mole-K } \mathrm{K}^{2} \\
\text { (Theory) }\end{array}$ & $\begin{array}{c}C_{\mathrm{es}} / T \\
\text { Joule/mole- } \mathrm{K}^{2} \\
\text { (Experimental) }\end{array}$ \\
\hline 1 & 80 & 1.3510 & 1.3520 \\
\hline 2 & 81 & 1.3635 & 1.3591 \\
\hline 3 & 82 & 1.3749 & 1.3650 \\
\hline 4 & 83 & 1.3866 & 1.3732 \\
\hline 5 & 84 & 1.3980 & 1.3803 \\
\hline 6 & 85 & 1.4106 & 1.3851 \\
\hline 7 & 86 & 1.4236 & 1.3945 \\
\hline 8 & 87 & 1.4372 & 1.4063 \\
\hline 9 & 87.9 & 1.4508 & 1.4170 \\
\hline
\end{tabular}

TABle 5: $\left(C-C_{\text {es }}\right) / T$ for $\mathrm{YBa}_{2} \mathrm{Cu}_{3} \mathrm{O}_{7-x}$ system.

\begin{tabular}{lccc}
\hline S. no. & $\begin{array}{c}\text { Temperature } \\
(\mathrm{K})\end{array}$ & $\begin{array}{c}\left(C-C_{\mathrm{es}}\right) / T \\
\text { oule/mole-K } \\
(\text { Theory })\end{array}$ & $\begin{array}{c}\left(C-C_{\mathrm{es}}\right) / T \\
\text { Joule/mole-K } \\
(\text { Experimental) }\end{array}$ \\
\hline 1 & 4 & 32.943048 & 8.542 \\
2 & 5 & 31.4759423 & 8.33 \\
3 & 6 & 30.4582297 & 7.92 \\
4 & 7 & 29.9338593 & 7.25 \\
5 & 8 & 29.6793776 & 7.083 \\
6 & 9 & 29.5322975 & 6.830 \\
7 & 10 & 29.398128 & 6.670 \\
8 & 11 & 29.2235587 & 6.670 \\
9 & 12 & 28.9786723 & 6.670 \\
10 & 13 & 28.6485315 & 6.670 \\
11 & 14 & 28.2270186 & 6.670 \\
12 & 15 & 27.7163961 & 6.670 \\
\hline
\end{tabular}

Using the following values

$$
\begin{gathered}
\omega_{n}=y \times 10^{-21} \mathrm{~J}, \quad \Delta=x \times 10^{-21} \mathrm{~J}, \quad l=1, \\
\omega_{0}=8 \times 10^{-21} \mathrm{~J}, \quad g^{2}=1 .
\end{gathered}
$$

The previous equation reduces as

$$
\frac{N(\omega)}{N(0)}=\frac{(y-8)}{\sqrt{(y-8)^{2}-x^{2}}}-\frac{(y+8)}{\sqrt{(y+8)^{2}-x^{2}}} .
$$

The values of density of states $N(\omega) / N(0)$ for the chosen value of $x=1.106,1.8851,2.334$ and 2.4761 are shown in Table 6 . The variation of density of states with $\omega$ at different temperatures is shown in Figure 5. 
TABLE 6: Density of states $N(\omega) / N(0)$ at various temperatures for $\mathrm{YBa}_{2} \mathrm{Cu}_{3} \mathrm{O}_{7-x}$ system.

\begin{tabular}{|c|c|c|c|c|c|}
\hline \multirow{2}{*}{ S. no. } & \multirow{2}{*}{ Frequency $(y)$} & \multicolumn{4}{|c|}{ Density of states } \\
\hline & & $\begin{array}{c}T=30 \mathrm{~K} \\
x=2.4761\end{array}$ & $\begin{array}{c}T=50 \mathrm{~K} \\
x=2.334\end{array}$ & $\begin{array}{c}T=70 \mathrm{~K} \\
x=1.8851\end{array}$ & $\begin{array}{c}T=85 \mathrm{~K} \\
x=1.106\end{array}$ \\
\hline 1 & 11.5 & 0.47791324 & 0.39120977 & 0.212929844 & 0.06485477 \\
\hline 2 & 12.0 & 0.303783239 & 0.256874065 & 0.149461031 & 0.04809573 \\
\hline 3 & 12.5 & 0.214803743 & 0.184567166 & 0.11137659 & 0.03708042 \\
\hline 4 & 13.0 & 0.161399908 & 0.139994146 & 0.0864163 & 0.02943323 \\
\hline 5 & 13.5 & 0.126234662 & 0.110167093 & 0.0690518 & 0.02390096 \\
\hline 6 & 14.0 & 0.101617339 & 0.089063119 & 0.056435285 & 0.01976737 \\
\hline 7 & 14.5 & 0.083611432 & 0.073510888 & 0.046958665 & 0.01659734 \\
\hline 8 & 15.0 & 0.069997004 & 0.061686732 & 0.03965028 & 0.01411343 \\
\hline 9 & 15.5 & 0.059430763 & 0.052471393 & 0.033891537 & 0.01213165 \\
\hline 10 & 16.0 & 0.051054964 & 0.045142493 & 0.029271859 & 0.01052594 \\
\hline 11 & 16.5 & 0.044298094 & 0.039214703 & 0.025509277 & 0.00920749 \\
\hline 12 & 17.0 & 0.038765958 & 0.034351053 & 0.022404542 & 0.00811226 \\
\hline 13 & 17.5 & 0.034178738 & 0.030311067 & 0.019813414 & 0.0071931 \\
\hline 14 & 18.0 & 0.030332928 & 0.026919081 & 0.017629272 & 0.00641463 \\
\hline
\end{tabular}

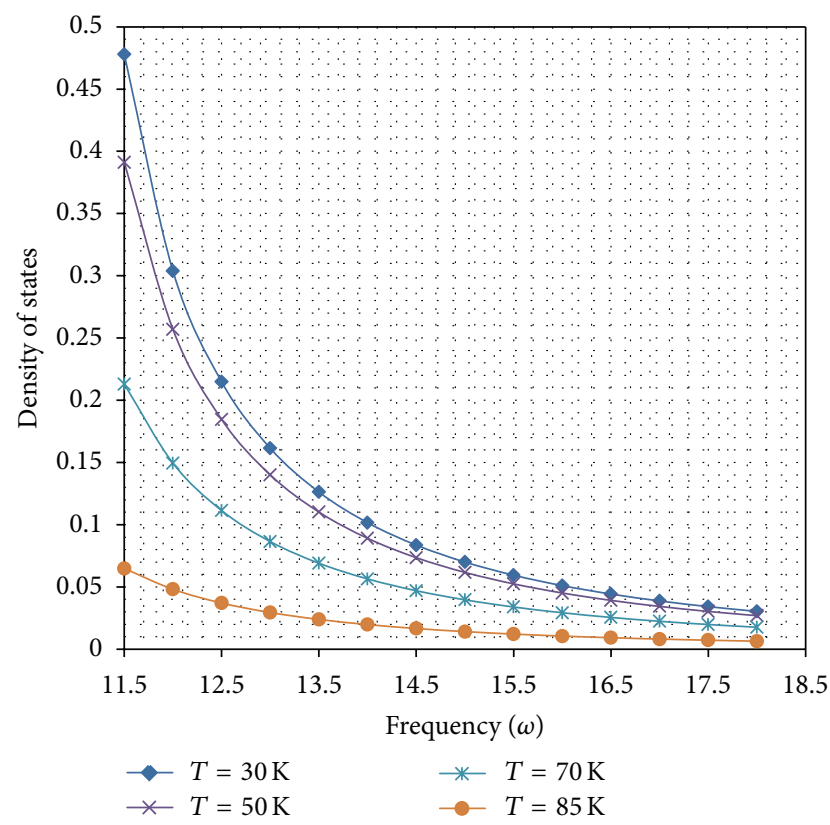

FIGURE 5: Variation of density of states with frequency $(\omega)$ at different temperatures for the system $\mathrm{YBa}_{2} \mathrm{Cu}_{3} \mathrm{O}_{7-x}$.

7.4. Free Energy Difference. The expression for the free energy difference can be expressed as

$$
\begin{aligned}
2\left[\frac{F_{S N}}{V}\right]_{\text {phonon }}=(-)[ & \frac{N(0) \Delta^{2}(k)}{2}-\frac{4 N(0)}{\beta} \\
& \times \frac{e^{-\beta \Delta(k)}}{2}\left(2 \pi \Delta(k) \beta^{-1}\right)^{1 / 2} \\
& \left.+\frac{4 N(0)}{\beta} \frac{1}{\beta}\left(\frac{\pi^{2}}{12}\right)\right] .
\end{aligned}
$$

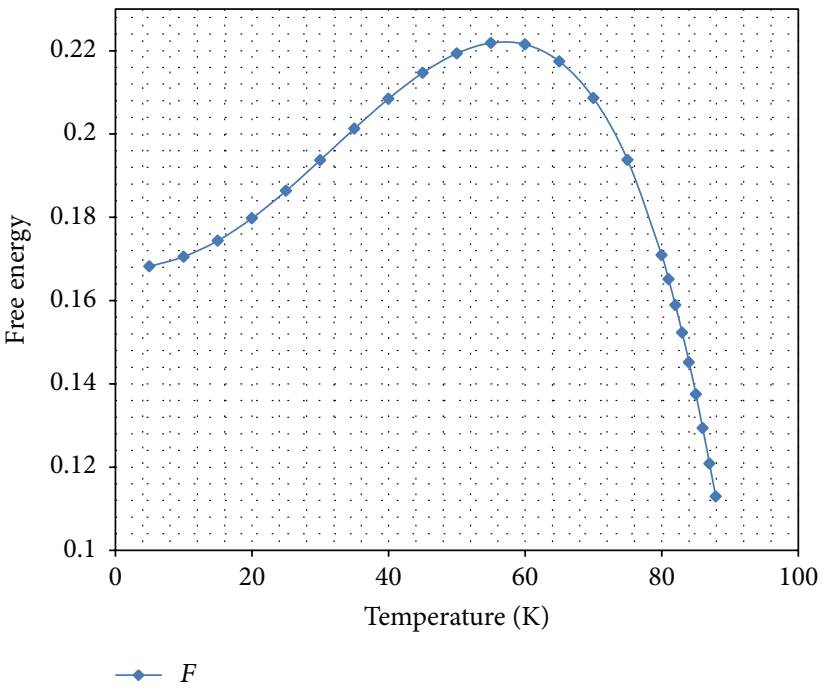

FIGURE 6: Variation of free energy difference with temperature for the system $\mathrm{YBa}_{2} \mathrm{Cu}_{3} \mathrm{O}_{7-x}$.

Solving numerically,

$$
\begin{aligned}
2\left[\frac{F_{S N}}{V}\right]_{\text {phonon }}= & (-) 0.02475 x^{2} \\
\times & {\left[1--0.01625\left[\frac{T}{x}\right]^{1 / 2} e^{-72.46 x / T}\right.} \\
& \left.+12.517 \times 10^{-4}\left[\frac{T}{x}\right]^{2}\right]
\end{aligned}
$$

The values of free energy difference at different temperatures are given in Table 7, and behaviour is shown in Figure 6, respectively. 
TABLE 7: Free energy difference for $\mathrm{YBa}_{2} \mathrm{Cu}_{3} \mathrm{O}_{7-x}$ system.

\begin{tabular}{lcc}
\hline S. no. & $\begin{array}{c}\text { Temperature } \\
(\mathrm{K})\end{array}$ & $\begin{array}{c}\text { Free energy difference } \\
\text { Joule/mole }\end{array}$ \\
\hline 1 & 5 & 0.1682 \\
2 & 10 & 0.1705 \\
3 & 15 & 0.1744 \\
4 & 20 & 0.1798 \\
5 & 25 & 0.1864 \\
6 & 30 & 0.1937 \\
7 & 35 & 0.2013 \\
8 & 40 & 0.2085 \\
9 & 45 & 0.2147 \\
10 & 50 & 0.2194 \\
11 & 55 & 0.2219 \\
12 & 60 & 0.2215 \\
13 & 65 & 0.2174 \\
14 & 70 & 0.2086 \\
15 & 75 & 0.1938 \\
16 & 80 & 0.1709 \\
17 & 85 & 0.1375 \\
18 & 86 & 0.1294 \\
19 & 87 & 0.1209 \\
20 & 87.9 & 0.1129 \\
\hline
\end{tabular}

7.5. Critical Field $\left(H_{c}\right)$. The low temperature critical field is given as

$$
\begin{aligned}
H_{c}=\{8 \pi( & \frac{N(0) \Delta^{2}(K)}{4}-\frac{4 N(0)}{\beta} \\
& \times \frac{e^{-\beta \Delta(\mathrm{K})}}{4}\left(\frac{2 \pi \Delta(\mathrm{K})}{\beta}\right)^{1 / 2} \\
& \left.\left.+\frac{4 N(0)}{\beta} \frac{1}{\beta}\left(\frac{\pi^{2}}{12}\right)\right)\right\}^{1 / 2} .
\end{aligned}
$$

Solving the previous equation numerically, we can obtain the values of low temperature critical field which are described in Table 8. The behavior of the low temperature critical field $\left(H_{c}\right)$ with temperature is shown in Figure 7.

\section{Discussion and Conclusions}

In the foregoing sections, we have developed the polaronic pairing mechanism of HTSC in cuprates and applied it to study physical properties of $\mathrm{YBa}_{2} \mathrm{Cu}_{3} \mathrm{O}_{7-x}$. The idea of polaron is based on the assumption about the autolocalization of an electron in the ion crystal due to its interaction with longitudinal optical vibrations under the local polarization, which is caused by the electron itself. The electron is confined to the local-polarization-induced potential well and

\begin{tabular}{|c|c|c|}
\hline S. no. & $\begin{array}{c}\text { Temperature } \\
(\mathrm{K})\end{array}$ & $\begin{array}{l}\text { Critical field } H_{C} \\
\text { Tesla } \\
\end{array}$ \\
\hline 1 & 5 & 1.454092 \\
\hline 2 & 10 & 1.46410 \\
\hline 3 & 15 & 1.480537 \\
\hline 4 & 20 & 1.503205 \\
\hline 5 & 25 & 1.530513 \\
\hline 6 & 30 & 1.560474 \\
\hline 7 & 35 & 1.590674 \\
\hline 8 & 40 & 1.618762 \\
\hline 9 & 45 & 1.642761 \\
\hline 10 & 50 & 1.660539 \\
\hline 11 & 55 & 1.669980 \\
\hline 12 & 60 & 1.668698 \\
\hline 13 & 65 & 1.653269 \\
\hline 14 & 70 & 1.619429 \\
\hline 15 & 75 & 1.560715 \\
\hline 16 & 80 & 1.465676 \\
\hline 17 & 81 & 1.440685 \\
\hline 18 & 82 & 1.413422 \\
\hline 19 & 83 & 1.383557 \\
\hline 20 & 84 & 1.351013 \\
\hline 21 & 85 & 1.314800 \\
\hline 22 & 86 & 1.275193 \\
\hline 23 & 87 & 1.232573 \\
\hline 24 & 87.9 & 1.191492 \\
\hline
\end{tabular}
conserves it by its own field [30].
TABLE 8: Critical field for $\mathrm{YBa}_{2} \mathrm{Cu}_{3} \mathrm{O}_{7-x}$ system.

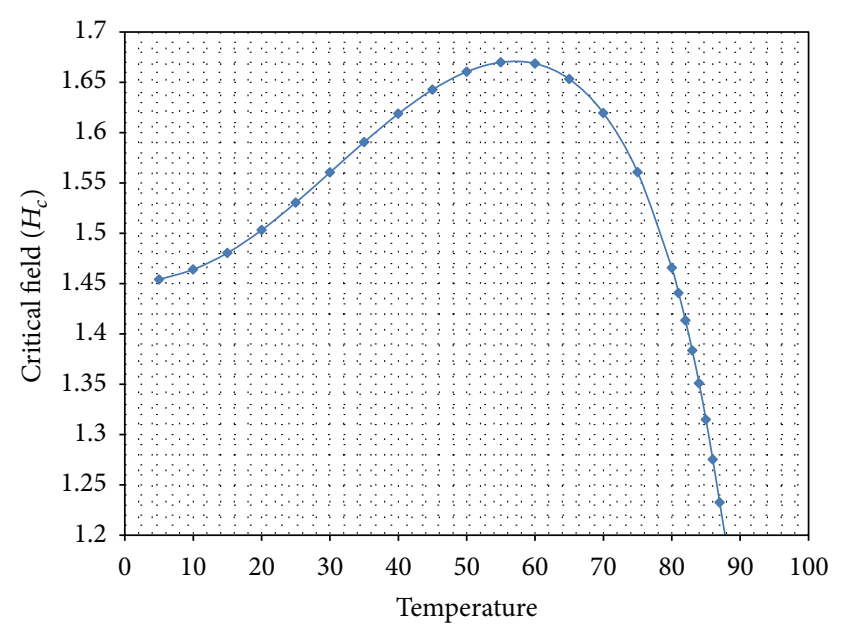

FIGURE 7: Variation of critical field with temperature for the system $\mathrm{YBa}_{2} \mathrm{Cu}_{3} \mathrm{O}_{7-x}$.

Following Green's function technique and equation of motion method, we have obtained expressions for superconducting order parameter. We found that phonon contribution is small enough. $T_{C}$ obtained for the system $\mathrm{YBa}_{2} \mathrm{Cu}_{3} \mathrm{O}_{7-x}$ is $88 \mathrm{~K}$ which is in a good agreement with experiments [1, 31]. Making use of various parameters given in Table 1, we have 
closely studied the density of states at various temperatures, specific heat, free energy, and critical field.

The specific heat behaviour with temperature is reasonably in a good agreement with experimental data [32].

The study of density of states, free energy, and critical field provides great insight into the physics of high- $T_{C}$ cuprates. In the absence of experimental results, we could not compare our results with experiments.

The investigations reported here aim to establish several of the properties of polaronic pairing mechanism of HTSC cuprates. Only a detailed comparison with future experiments will clarify whether this pairing mechanism is or not a good approximation to describe HTSC cuprates.

\section{References}

[1] T. Tohyama, "Recent progress in physics of high-temperature superconductors," Japanese Journal of Applied Physics, vol. 51, no. 1, Article ID 010004, 2012.

[2] S. Kruchinin, H. Nagao, and S. Aono, Modern Aspects of Superconductivity. Theory of Superconductivity, World Scientific, River Edge, NJ, USA, 2011.

[3] S. L. Kakani and S. Kakani, Superconductivity, Anshan, Kent, UK, 2009.

[4] K. P. Sinha and S. L. Kakani, High Temperature Superconductivity: Current Results \& Novel Mechanisms, Nova Science, New York, NY, USA, 1995.

[5] K. P. Sinha and S. L. Kakani, "Fermion local charged Boson model and cuprate superconductors," Proceedings of the National Academy of Sciences, vol. 72, pp. 153-214, 2002.

[6] K. H. Bennemann and J. B. Ketterson, Superconductivity: Conventional and Unconventional Superconductors, Vol. 1, 2, Springer, New York, NY, USA, 2008.

[7] J. R. Schrieffer, Theory of Superconductivity, Westview Press, Oxford, UK, 1999.

[8] M. R. Norman, "Cuprates-an overview," Journal of Superconductivity and Novel Magnetism, vol. 25, no. 7, pp. 2131-2134, 2012.

[9] A. S. Alexandrov, Theory of Superconductivity from Weak to Strong Coupling, IOP publishing, Bristol, UK, 2003.

[10] N. Plakida, High Temperature Cuprate Superconductors: Experiment, Theory and Applications, Springer, Heidelberg, Germany, 2010.

[11] I. Askerzade, Unconventional Superconductors, vol. 153 of Springer Series in Material Science, Springer, Berlin, Germany, 2012.

[12] O. Gunnarsson and O. Rösch, "Interplay between electronphonon and Coulomb interactions in cuprates ," Journal of Physics, vol. 20, no. 4, Article ID 043201, 2008.

[13] J. Bardeen, L. N. Cooper, and J. R. Schrieffer, "Theory of superconductivity," Physical Review, vol. 108, no. 5, pp. 11751204, 1957.

[14] G. M. Eliashberg, Zhurnal Eksperimental'noi i Teoreticheskoi Fiziki, vol. 38, p. 966, 1960, Engkish translation in The Journal of Experimental and Theoretical Physics: Soviet Physics, vol. 11, p. 696, 1960

[15] W. L. McMillan and J. M. Rowell, "Lead phonon spectrum calculated from superconducting density of states," Physical Review Letters, vol. 14, pp. 108-112, 1965.

[16] W. L. McMillan, "Transition temperature of strong-coupled superconductors," Physical Review, vol. 167, no. 2, pp. 331-344, 1968.
[17] P. Brusov, Mechanisms of High Temperature Superconductivity, Rostov State University Publishing, 1999.

[18] T. Morya and K. Ueda, "Spin fluctuations and high-temperature superconductivity," Advances in Physics, vol. 49, no. 5, pp. 556606, 2000.

[19] D. Manske, Theory of Unconventional Superconductors, Springer, Heidelberg, Germany, 2004.

[20] A. Alexandrov and J. Ranninger, "Theory of bipolarons and bipolaronic bands," Physical Review B, vol. 23, no. 4, pp. 17961801, 1981.

[21] A. S. Alexandrov and J. Ranninger, "Photoemission spectroscopu of the superconducting and normal state for polaronic systems," Physica C, vol. 198, no. 3-4, pp. 360-370, 1992.

[22] T. Holstein, "Studies of polaron motion: part I. The molecularcrystal model," Annals of Physics, vol. 8, pp. 325-342, 1959.

[23] L. G. Lang and Y. A. Firsov, Zhurnal Eksperimental'Noi $i$ TeoreticheskoI Fiziki, vol. 43, p. 1843, 1962.

[24] I. L. G. Lang and Y. A. Firsov, "Kinetic theory of semiconductors with low mobility," Journal of Experimental and Theoretical Physics: Soviet Physics, vol. 16, p. 1301, 1963.

[25] G. D. Mahan, Many Particle Physics, Plenum Press, New York, NY, USA, 1982.

[26] D. N. Zubaroev, "Double-time green functions in statistical physics," Uspekhi Fizicheskikh Nauk, vol. 71, pp. 71-116, 1960.

[27] A. L. Fetter and J. D. Walecka, Quantam Theory of Many Particle System, McGraw-Hill, New York, NY, USA, 1971.

[28] S. C. Tiwari, A. Kashyap, A. K. Surana, R.K. Paliwal, and S. L. KaKani, "polaronic mechanism of superconductivity in doped fulleride systems," International Journal of Modern Physics B, vol. 23, p. 615, 2009.

[29] R. Lortz, T. Tomita, Y. Wang et al., "On the origin of the double superconducting transition in overdoped $\mathrm{YBa}_{2} \mathrm{Cu}_{3} \mathrm{O}_{x}$," Physica C, vol. 434, pp. 194-198, 2006.

[30] S. I. Pekar, Studies on the Electron Theory of Crystals, Gostekhizdat, Moscow, Russia, 1951.

[31] B. J. Ramshaw, J. Day, B. Vignolle et al., "Vortex lattice melting and $\mathrm{H}_{c 2}$ in underdoped $\mathrm{YBa}_{2} \mathrm{Cu}_{3} \mathrm{O}_{y}$," Physical Review $B$, vol. 86, no. 17, Article ID 174501, 6 pages, 2012.

[32] M. J. W. Dodgson, V. B. Geshkenbein, H. Nordborg, and G. Blatter, "Thermodynamics of the first-order vortex lattice melting transition in $\mathrm{YBa}_{2} \mathrm{Cu}_{3} \mathrm{O}_{7-\delta}$," Physical Review B, vol. 57, no. 22, pp. 14498-14506, 1998. 

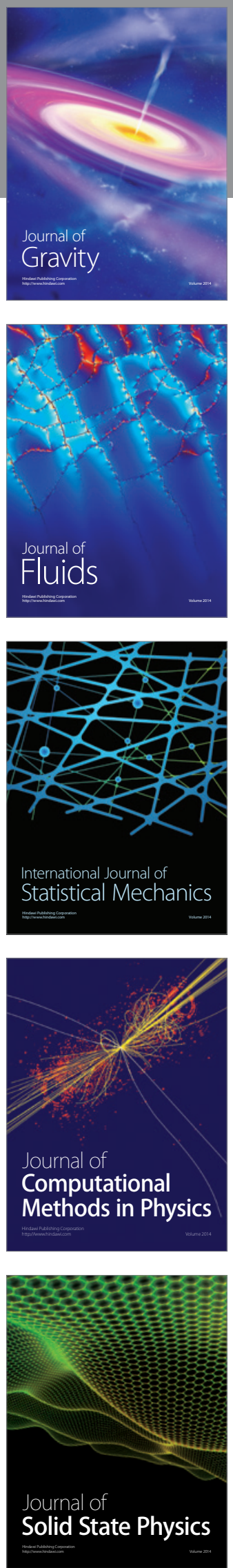

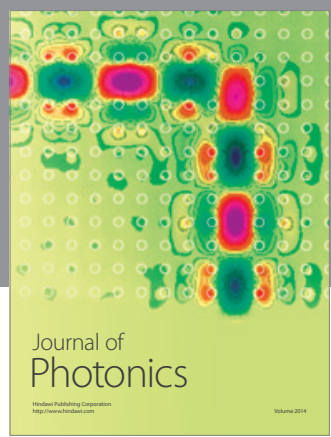

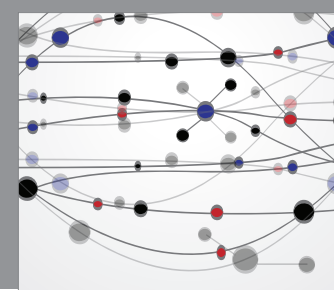

The Scientific World Journal

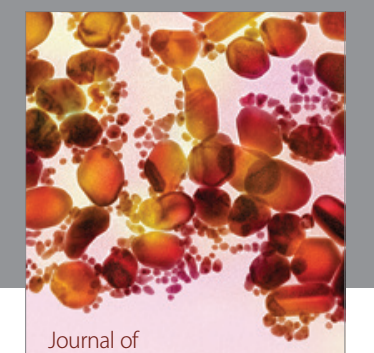

Soft Matter
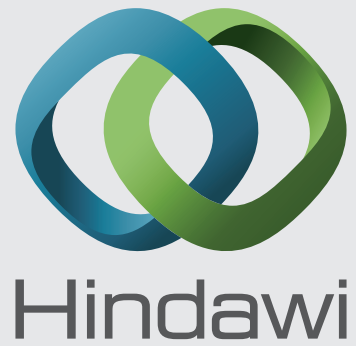

Submit your manuscripts at

http://www.hindawi.com
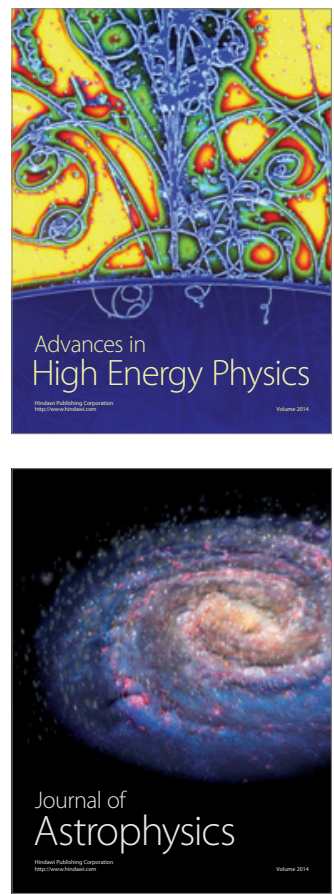
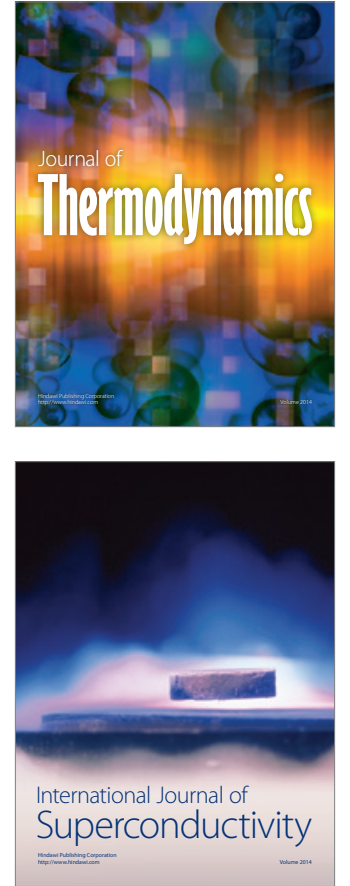
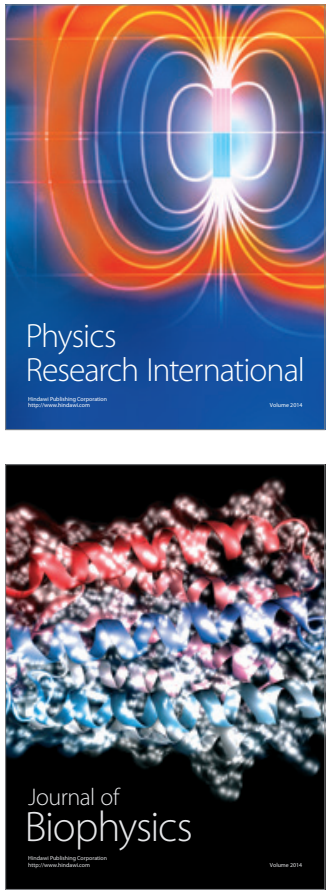
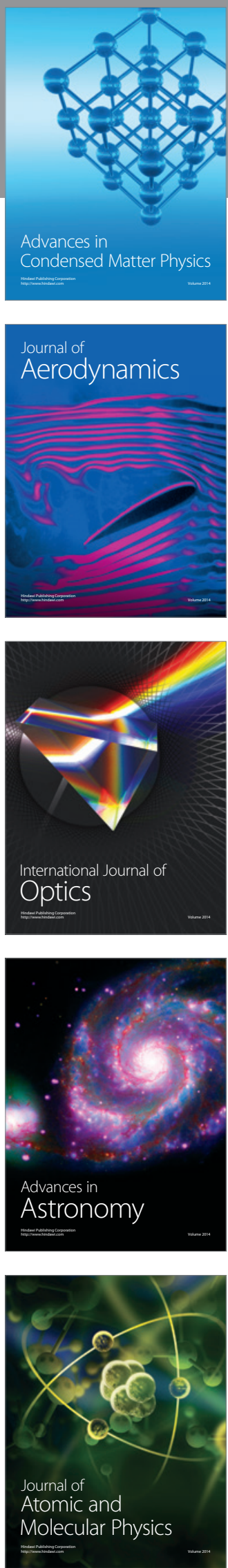